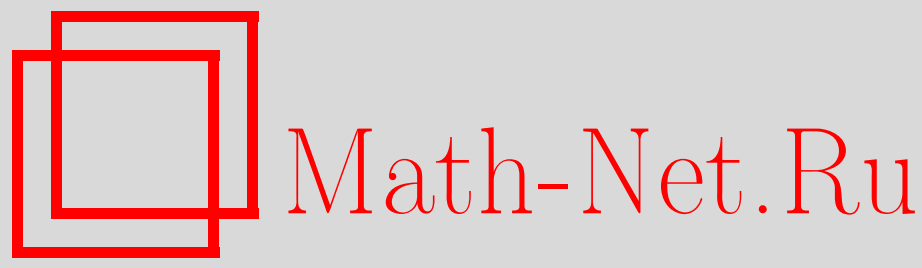

О. С. Круглинская, Корреляционные функции и спектральные кривые в моделях минимальной гравитации, TMФ, 2013, том 174, номер 1, 90-98

DOI: https://doi.org/10.4213/tmf8388

Использование Общероссийского математического портала Math-Net.Ru подразумевает, что вы прочитали и согласны с пользовательским соглашением http://www . mathnet.ru/rus/agreement

Параметры загрузки:

IP: 54.198 .55 .26

26 апреля 2023 г., $15: 45: 48$

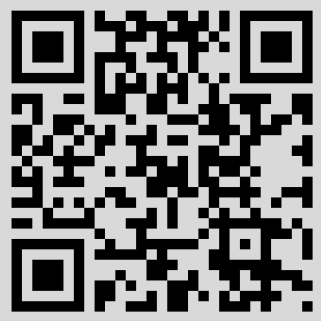




\title{
ФИЗИКА
}

Том 174, № 1

январь, 2013

(C) 2013 г.

\author{
О. С. Круглинская*
}

\section{КОРРЕЛЯЦИОННЫЕ ФУНКЦИИ И СПЕКТРАЛЬНЫЕ КРИВЫЕ В МОДЕЛЯХ МИНИМАЛЬНОЙ ГРАВИТАЦИИ}

\begin{abstract}
Обсуждаются корреляторы для моделей минимальной гравитации. Предложен алгоритм для вычисления инвариантных отношений из формул вычетов, которые можно сравнивать с коэффициентами разложения статистической суммы в теории Лиувилля. Для моделей серии $(2,2 K-1)$ явно получены коэффициенты перехода от базиса квазиклассической иерархии к базису теории Лиувилля, а также проверена непосредственным вычислением гипотеза о форме спектральной кривой.
\end{abstract}

Ключевые слова: производящие функции, теория Лиувилля, интегрируемые системы, корреляционные функции.

DOI: $10.4213 / \operatorname{tmf} 8388$

\section{1. ВВЕДЕНИЕ}

Хорошо известно, что матричные модели описывают двумерную квантовую гравитацию с центральным зарядом $c \leqslant 1$. В общем случае для описания теории двумерной гравитации с использованием $(1 / N)$-разложения [1] необходимо учитывать струнные петли - мировые листы рода больше нуля. Мы будем рассматривать только предел $N \rightarrow \infty$, отвечающий вкладу поверхностей рода нуль.

Статистическую сумму одноматричной модели можно представить в виде интеграла по собственным значениям (см., например, обзор [2] и библиографию в нем):

$$
Z=\int d \Phi e^{-(1 / \hbar) \operatorname{Tr} W(\Phi)}=\frac{1}{N !} \int \prod_{i=1}^{N}\left(d \phi_{i} e^{-(1 / \hbar) W\left(\phi_{i}\right)}\right) \Delta^{2}(\phi) .
$$

В этом выражении $\Phi$ является матрицей размера $N \times N, \hbar=t_{0} / N$ - параметр квазиклассического разложения (величина $t_{0}$ остается фиксированной и конечной при $N \rightarrow \infty, \hbar \rightarrow 0)$, а $W(\Phi)$ в общем случае определяется формулой

$$
W(\Phi)=\sum_{k>0} t_{k} \Phi^{k}
$$

где $t_{k}$ - источники, a $\operatorname{tr} \Phi^{k}-$ некоторый базис в пространстве операторов.

* Физический институт им. П. Н. Лебедева РАН, Москва, Россия.

E-mail: kruglinskaya@gmail.com 
В меру интегрирования в правой части выражения (1) входит определитель Вандермонда

$$
\Delta(\phi)=\prod_{i<j}\left(\phi_{i}-\phi_{j}\right)
$$

Свободная энергия $F=\ln Z$ может быть представлена через разложение в ряд по четным степеням $\hbar$, которое будет эквивалентно разложению по обратному размеру матриц:

$$
F\left(t_{0} \mid\left\{t_{k}\right\}\right)=\sum_{g=0}^{\infty} \hbar^{2 g-2} F_{g}\left(t_{0} \mid\left\{t_{k}\right\}\right), \quad N \rightarrow \infty, \quad \hbar \rightarrow 0,
$$

когда $N \hbar=t_{0}$ фиксировано.

В случае больших $N$ основной вклад вносит только первое слагаемое $F_{0}$ рода нуль. Следовательно, будем учитывать только корреляционные функции на сфе-

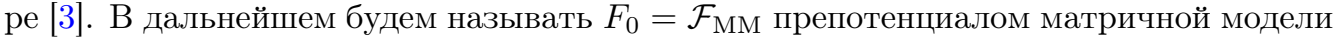
по аналогии с топологическими струнами и суперсимметричными калибровочными теориями.

Вообще говоря, системой Зайберга-Виттена, определяющей препотенциал $\mathcal{F}$, называется следующий набор данных [4], [5]:

- семейство $\mathcal{M}$ римановых поверхностей (комплексных кривых) $\mathcal{C}$, размерность пространства модулей которого совпадает с родом кривой $\mathcal{C}$;

- мероморфный дифференциал $d S$, вариации которого относительно модулей кривых голоморфны.

В переменных

$$
a_{i} \equiv \oint_{A_{i}} d S
$$

препотенциал $\mathcal{F}$ определяется из выражений

$$
\frac{\partial \mathcal{F}}{\partial a_{i}} \equiv \oint_{B_{i}} d S
$$

Интегрирование в $(5)$ и $(6)$ ведется по $A$ - и $B$-циклам, а формула $(6)$ определяет $\mathcal{F}$ в силу билинейных соотношений Римана.

Прямыми аналогами выражений (5), (6) являются формулы вычетов (см. [3], [6]):

$$
\begin{aligned}
t_{k} & =\frac{1}{k} \operatorname{res}_{\infty} \xi^{-k} d S, & & k>0, \\
\frac{\partial \mathcal{F}_{\mathrm{MM}}}{\partial t_{k}} & =\operatorname{res}_{\infty} \xi^{k} d S, & & k>0,
\end{aligned}
$$

определяющие некоторым образом препотенциал матричной модели $F_{0}=\mathcal{F}_{\mathrm{MM}}$, где $\xi$ - обратная локальная координата.

Уравнение спектральной кривой $\mathcal{C}$ в случае одноматричной модели (1)

$$
y^{2}=W^{\prime 2}(x)+4 f(x)
$$

может быть получено из петлевого уравнения при $N \rightarrow \infty$ [7]. Оно описывает гиперэллиптическую кривую, в терминах которой может быть полностью определена свободная энергия $F_{0}=\mathcal{F}_{\mathrm{MM}}$ при $d S=y d x$. 
В уравнении (9) $y=W^{\prime}(x)-2 G$, а $G$ - производящая функция (резольвента):

$$
G(x)=\left\langle\operatorname{Tr} \frac{1}{x-\Phi}\right\rangle=\left\langle\sum_{i=1}^{N} \frac{1}{x-\phi_{i}}\right\rangle,
$$

$f(x)$ - полином степени на 2 меньшей, чем степень $W(x)$, определенный из асимптотики $x \rightarrow \infty$.

\section{2. МОДЕЛИ МИНИМАЛЬНОЙ \\ ГРАВИТАЦИИ: $(p, q)$ КРИТИЧЕСКИЕ ТОЧКИ}

Считая матричные модели дискретным аналогом двумерной гравитации, статистическую сумму последней в этой гипотезе можно получить из статистической суммы матричной модели в результате некоторого нетривиального и сингулярного преобразования [8]. Детали этого предельного перехода были изучены в работе [9], мы же приводим результаты.

Минимальные конформные теории [10], взаимодействующие с лиувиллевской гравитацией [11], называются $(p, q)$-моделями минимальной гравитации. Чтобы вычислить статистическую сумму $(p, q)$-моделей для рода нуль, используется тот же метод, что и для вычисления препотенциала матричной модели. Переменные $t_{k}$, также называемые временами, и свободную энергию $\mathcal{F}$ в неявном виде можно записать, используя формулы (7), (8), в которых теперь

$$
d S=Y d X, \quad \xi=X^{1 / p} .
$$

Для любой $(p, q)$-точки берется пара полиномов

$$
X=\lambda^{p}+x_{p-1} \lambda^{p-1}+\cdots, \quad Y=\lambda^{q}+y_{q-1} \lambda^{q-1}+\cdots,
$$

которые удовлетворяют уравнению плоской кривой вида

$$
Y^{p}=X^{q}+\sum C_{i j} X^{i} Y^{j}
$$

Если зафиксировать $p=2, q=2 K-1$, то

$$
Y^{2}=P_{2 K-1}(X)
$$

Последнее уравнение в случае общего положения описывает риманову поверхность рода $g=K-1$ с $2 K$ точками ветвления: корни полинома $P_{2 K-1}(X)=0$ плюс $X=Y=\infty$.

Согласно гипотезе, предложенной в работе [12], уравнение кривой (14) для минимальной гравитации с точностью до коэффициента совпадает с уравнением на полиномы Чебышева. В настоящей работе это предположение конкретизировано и проверено для ряда моделей прямым вычислением.

Для сравнения с теорией Лиувилля необходимо учитывать неоднозначный выбор базиса, обусловленный тем, что времена имеют скейлинговую размерность, которая может совпадать с суммой размерностей других времен в той же модели:

$$
\begin{gathered}
t_{k} \rightarrow t_{k}+A t_{\alpha} t_{\beta}, \quad A=\text { const }, \\
\Delta_{k}=\Delta_{\alpha}+\Delta_{\beta}, \quad \alpha, \beta<k .
\end{gathered}
$$


В этом случае имеет место резонанс [13], [14]. В формуле (16) $\Delta_{k}=p+q-k-$ скейлинговая размерность времени $t_{k}$, а $\Delta_{\alpha}, \Delta_{\beta}$ - размерности $t_{\alpha}$ и $t_{\beta}$ соответственно.

В моделях минимальной гравитации $(2,2 K-1)$ космологическая постоянная $\mu=t_{2 K-3}$ всегда имеет размерность $\Delta_{\mu}=4$. Тогда резонансы с космологической постоянной

$$
t_{2 K-3-4 i} \rightarrow t_{2 K-3-4 i}+A_{i} \mu^{i+1}, \quad i=1,2, \ldots .
$$

Коэффициенты $A_{i}$ можно найти из условия $\partial \mathcal{F} / \partial t_{i}=0$ для одноточечных функций и струнного уравнения.

Далее мы описываем схему вычислений коэффициентов перехода $\left\{A_{i}\right\}$ для модели $(2,9)$. В приложении приведены вычисленные коэффициенты для случаев $K=$ $6,7,8,9,10,11,12$.

\section{3. ПРИМЕР: МОДЕЛЬ $(2,9)$}

Для полиномов (12) в этом случае

$$
X=\lambda^{2}+x_{0}, \quad Y=\lambda^{9}+y_{7} \lambda^{7}+y_{5} \lambda^{5}+y_{3} \lambda^{3}+y_{1} \lambda .
$$

Вычисляем времена через формулы (7):

$$
\begin{gathered}
t_{1}=-x_{0} y_{1}+\frac{3}{4} x_{0}^{2} y_{3}-\frac{5}{8} x_{0}^{3} y_{5}+\frac{35}{64} x_{0}^{4} y_{7}-\frac{63}{128} x_{0}^{5}, \\
t_{3}=\frac{2}{3} y_{1}-x_{0} y_{3}+\frac{5}{4} x_{0}^{2} y_{5}-\frac{35}{24} x_{0}^{3} y_{7}+\frac{105}{64} x_{0}^{4}, \\
t_{5}=\frac{2}{5} y_{3}-x_{0} y_{5}+\frac{7}{4} x_{0}^{2} y_{7}-\frac{21}{8} x_{0}^{3}, \\
t_{7}=\frac{2}{7} y_{5}-x_{0} y_{7}+\frac{9}{4} x_{0}^{2}, \quad t_{9}=\frac{2}{9} y_{7}-x_{0}=0, \quad t_{11}=\frac{2}{11} .
\end{gathered}
$$

Решая последние пять уравнений, линейных относительно $y$, и подставляя решения в уравнение для $t_{1}$, получаем струнное уравнение

$$
t_{1}+\frac{3}{2} x_{0} t_{3}+\frac{63}{128} x_{0}^{5}+\frac{15}{8} x_{0}^{2} t_{5}+\frac{35}{16} x_{0}^{3} t_{7}=0 .
$$

В этой модели возможен только один резонанс:

$$
t_{3} \rightarrow t_{3}+A_{3} \mu^{2}, \quad \mu=t_{7} .
$$

Из условия обращения в ноль одноточечной функции $\partial \mathcal{F} / \partial t_{3}$ и струнного уравнения находим космологическую постоянную и константу $A_{3}$ :

$$
\mu=-\frac{9}{28} x_{0}^{2}, \quad A_{3}=\frac{49}{36} .
$$

Свободную энергию $\mathcal{F}$ моделей минимальной гравитации $(2,2 K-1)$ можно записать в виде

$$
\mathcal{F}\left(t_{i}\right)=\mu^{K+1 / 2} f\left(\tau_{j}\right),
$$

где $f$ - масштабно-инвариантная функция, $\tau_{j}$ - безразмерные отношения времен. 
В данном примере

$$
\begin{gathered}
\mathcal{F}\left(t_{1}, t_{3}, t_{5}, \mu\right)=\mu^{11 / 2} f\left(\frac{t_{1}}{\mu^{5 / 2}}, \frac{t_{3}}{\mu^{2}}, \frac{t_{5}}{\mu^{3 / 2}}\right), \quad \frac{\partial \mathcal{F}}{\partial t_{1}}=\mu^{3} f^{(1)}, \\
\frac{\partial^{2} \mathcal{F}}{\partial t_{1}^{2}}=\mu^{1 / 2} f^{(11)}=\frac{x_{0}}{2}, \quad \ldots,
\end{gathered}
$$

а безразмерные отношения

$$
\tau_{1}=\frac{t_{1}}{\mu^{5 / 2}}, \quad \tau_{2}=\frac{t_{3}}{\mu^{2}}, \quad \tau_{3}=\frac{t_{5}}{\mu^{3 / 2}} .
$$

Перепишем струнное уравнение $u=f^{(11)}$ в безразмерных терминах:

$$
\tau_{1}+3 u \tau_{2}+\frac{63}{4} u^{5}+\frac{15}{2} u^{2} \tau_{3}+\frac{35}{2} u^{3}=0 .
$$

Разложим функцию $f$ в ряд:

$$
\begin{aligned}
f=f_{0} & +f_{1} \tau_{1}+f_{2} \tau_{2}+f_{3} \tau_{3}+\frac{1}{2} f_{11} \tau_{1}^{2}+\frac{1}{2} f_{22} \tau_{2}^{2}+\frac{1}{2} f_{33} \tau_{3}^{2}+f_{12} \tau_{1} \tau_{2}+ \\
& +f_{23} \tau_{2} \tau_{3}+f_{13} \tau_{1} \tau_{3}+\frac{1}{6} f_{111} \tau_{1}^{3}+\frac{1}{6} f_{222} \tau_{2}^{3}+\frac{1}{6} f_{333} \tau_{3}^{3}+ \\
& +\frac{1}{2} f_{123} \tau_{1} \tau_{2} \tau_{3}+\frac{1}{2} f_{112} \tau_{1}^{2} \tau_{2}+\frac{1}{2} f_{122} \tau_{2}^{2} \tau_{1}+\cdots
\end{aligned}
$$

Аналитические члены $f_{1}$ и $f_{3}$ в этом разложении не представляют интереса, так как они не универсальны.

Из уравнения (25) можно найти старшие коэффициенты при $\tau_{2}=0, \tau_{3}=0$ :

$$
f_{111}=-\frac{4}{105 f_{11}^{2}\left(3 f_{11}^{2}+2\right)}, \quad f_{112}=-\frac{3}{63 f_{11}^{3}+35 f_{11}}, \quad \ldots
$$

В результате, используя (8), (23) и (26), получаем безразмерные отношения, например

$$
\frac{f_{1111} f_{11}}{f_{111}^{2}}=-7, \quad \frac{f_{1111} f_{22}}{f_{112}^{2}}=-\frac{7}{3} .
$$

Аналогично можно легко вычислить отношения для других $(2,2 K-1)$-моделей. Например, для модели $(2,11)$ с производящей функцией

$$
\mathcal{F}=\mu^{13 / 2} \tilde{f}\left(\tilde{t_{1}} / \mu^{3}, \tilde{t_{3}} / \mu^{5 / 2}, \tilde{t_{5}} / \mu^{2}, \tilde{t_{7}} / \mu^{3 / 2}\right)
$$

мы имеем

$$
\frac{\tilde{f}_{1111} \tilde{f}_{11}}{\tilde{f}_{111}^{2}}=-9, \quad \frac{\tilde{f}_{1111} \tilde{f}_{22}}{\tilde{f}_{112}^{2}}=-3 .
$$

Именно безразмерные отношения (27), (28) имеет смысл сравнивать с результатами вычислений в теории Лиувилля, полученными в последние годы, используя высшие уравнения Лиувилля [15]. 


\section{4. УРАВНЕНИЕ СПЕКТРАЛЬНОЙ КРИВОЙ}

Интересующее нас уравнение спектральной кривой можно получить, используя формулы (12), где $y_{i}$ выражены через $t_{k}$ с помощью (7). При этом стоит помнить, что в базисе теории Лиувилля учитываются резонансы времен $t_{k}$ с космологической постоянной $\mu$ (формула $(17))$, после чего $t_{k}$ полагаются равными нулю.

Например, запишем уравнение кривой для модели $(2,9)$, содержащее только $\mu$ и $t_{3}$. Для этого выразим из первого уравнения (18) $\lambda$ и подставим в выражение для $Y$. В свою очередь, все $y_{i}$ выражаются через времена (19). Производя замену $X^{\prime}=-X / x_{0}$, получаем

$$
\begin{aligned}
Y^{2} & =\frac{1}{126}\left(X-x_{0}\right)\left(x_{0}^{4}-4 X x_{0}^{3}-12 X^{2} x_{0}^{2}+8 X^{3} x_{0}+16 X^{4}\right)^{2}= \\
& =\frac{1}{126 x_{0}^{9}}\left(X^{\prime}+1\right)\left(2 X^{\prime}-1\right)^{2}\left(8 X^{\prime 3}-6 X^{\prime}-1\right)^{2} .
\end{aligned}
$$

Заметим, что правая часть этого равенства выражается через полином Чебышева $T_{9}(a)$ после замены $a=\sqrt{\left(X^{\prime}+1\right) / 2}$ :

$$
\begin{aligned}
T_{9}^{2} & =\left(256 a^{9}-576 a^{7}+432 a^{5}-120 a^{3}+9 a\right)^{2}= \\
& =\frac{1}{2}\left(X^{\prime}+1\right)\left(2 X^{\prime}-1\right)^{2}\left(8 X^{\prime 3}-6 X^{\prime}-1\right)^{2},
\end{aligned}
$$

последнее выражение с точностью до коэффициента совпадает с уравнением кривой

$$
Y^{\prime}=T_{9}(a), \quad X^{\prime}=T_{2}(a)=2 a^{2}-1
$$

и соответствует гипотезе Зайберга-Шиха [12].

Аналогичным образом можно проверить гипотезу для всех $(2,2 K-1)$-моделей, некоторые из которых представлены в приложении.

\section{5. ЗАКЛЮЧЕНИЕ}

В настоящей работе предложен алгоритм вычисления корреляторов - коэффициентов разложения производящих функций в моделях $(2,2 K-1)$ в правильном базисе с точки зрения непрерывной двумерной гравитации [13]. Безразмерные отношения этих коэффициентов можно связать с результатами теории Лиувилля, вычисления в которой гораздо сложнее (см., например, работу [15]).

Также получены точные уравнения спектральных кривых в лиувиллевском базисе. Прямым вычислением проверена гипотеза, согласно которой уравнения кривых с точностью до коэффициента совпадают с уравнениями для полиномов Чебышева.

Отметим, что для общего случая $(p, q)$-моделей минимальной гравитации с произвольными $p$ и $q$ алгоритм перехода к базису Лиувилля по-прежнему неизвестен. Некоторые соображения на этот счет приведены, например, в работах [14], [16]. 
ПРИЛОЖЕНИЕ

\begin{tabular}{|c|c|c|}
\hline$(2,2 K-1)$ & $\begin{array}{c}\text { Резонансы } \\
\text { с космологической } \\
\text { постоянной }\end{array}$ & Струнное уравнение и уравнение кривой \\
\hline$(2,11)$ & $\begin{array}{l}t_{1}=\frac{729}{968} \mu^{3} \\
t_{5}=\frac{81}{44} \mu^{2} \\
\mu=-\frac{11}{36} x_{0}^{2}\end{array}$ & $\begin{aligned} t_{1}+ & \frac{15}{8} x_{0}^{2} t_{5}+\frac{315}{128} x_{0}^{4} t_{9}+\frac{231}{512} x_{0}^{6}+\frac{35}{16} x_{0}^{3} t_{7}+\frac{3}{2} x_{0} t_{3}=0 \\
Y^{2}= & \frac{1}{1024}\left(X-x_{0}\right)\left(x_{0}^{5}+6 X x_{0}^{4}-12 X^{2} x_{0}^{3}-\right. \\
& \left.-32 X^{3} x_{0}^{2}+16 X^{4} x_{0}+32 X^{5}\right)^{2}\end{aligned}$ \\
\hline$(2,13)$ & $\begin{aligned} t_{3} & =\frac{6655}{4056} \mu^{3} \\
t_{7} & =\frac{121}{52} \mu^{2} \\
\mu & =-\frac{13}{44} x_{0}^{2}\end{aligned}$ & $\begin{aligned} t_{1}+ & \frac{3}{2} x_{0} t_{3}+\frac{693}{256} x_{0}^{5} t_{11}+\frac{429}{1024} x_{0}^{7}+ \\
& +\frac{15}{8} x_{0}^{2} t_{5}+\frac{35}{16} x_{0}^{3} t_{7}+\frac{315}{128} x_{0}^{4} t_{9}=0 \\
Y^{2}= & \frac{1}{4096}\left(X-x_{0}\right)\left(32 X^{5} x_{0}-80 X^{4} x_{0}^{2}-32 X^{3} x_{0}^{3}+\right. \\
& \left.+24 X^{2} x_{0}^{4}+6 X x_{0}^{5}+64 X^{6}-x_{0}^{6}\right)^{2}\end{aligned}$ \\
\hline$(2,15)$ & $\begin{aligned} t_{1} & =\frac{28561}{43200} \mu^{4} \\
t_{5} & =\frac{15379}{5400} \mu^{3} \\
t_{9} & =\frac{169}{60} \mu^{2} \\
\mu & =-\frac{15}{52} x_{0}^{2}\end{aligned}$ & $\begin{aligned} t_{1}+ & \frac{6435}{16384} x_{0}^{8}+\frac{3003}{1024} x_{0}^{6} t_{13}+\frac{693}{256} x_{0}^{5} t_{11}+ \\
& +\frac{315}{128} x_{0}^{4} t_{9}+\frac{3}{2} x_{0} t_{3}+\frac{35}{16} x_{0}^{3} t_{7}+\frac{15}{8} x_{0}^{2} t_{5}=0, \\
Y^{2}= & \frac{1}{16384}\left(X-x_{0}\right)\left(128 X^{7}-x_{0}^{7}+64 X^{6} x_{0}-192 X^{5} x_{0}^{2}-\right. \\
& \left.-80 X^{4} x_{0}^{3}+80 X^{3} x_{0}^{4}+24 X^{2} x_{0}^{5}-8 X x_{0}^{6}\right)^{2}\end{aligned}$ \\
\hline$(2,17)$ & $\begin{aligned} t_{3} & =\frac{590625}{314432} \mu^{4} \\
t_{7} & =\frac{10125}{2312} \mu^{3} \\
t_{11} & =\frac{225}{68} \mu^{2} \\
\mu & =-\frac{17}{60} x_{0}^{2}\end{aligned}$ & $\begin{aligned} t_{1}+ & \frac{6435}{2048} x_{0}^{7} t_{15}+\frac{3003}{1024} x_{0}^{6} t_{13}+\frac{693}{256} x_{0}^{5} t_{11}+\frac{315}{128} x_{0}^{4} t_{9}+ \\
& +\frac{35}{16} x_{0}^{3} t_{7}+\frac{15}{8} x_{0}^{2} t_{5}+\frac{3}{2} x_{0} t_{3}+\frac{12155}{32768} x_{0}^{9}=0 \\
Y^{2}= & \frac{1}{65536}\left(X-x_{0}\right)\left(256 X^{8}+x_{0}^{8}+128 X^{7} x_{0}-448 X^{6} x_{0}^{2}-\right. \\
& \left.-192 X^{5} x_{0}^{3}+240 X^{4} x_{0}^{4}+80 X^{3} x_{0}^{5}-40 X^{2} x_{0}^{6}-8 X x_{0}^{7}\right)^{2}\end{aligned}$ \\
\hline$(2,19)$ & $\begin{array}{c}t_{1}=\frac{9938999}{16681088} \mu^{5} \\
t_{5}=\frac{1753941}{438976} \mu^{4} \\
t_{9}=\frac{54043}{8664} \mu^{3} \\
t_{13}=\frac{289}{76} \mu^{2} \\
\mu=-\frac{19}{68} x_{0}^{2}\end{array}$ & $\begin{aligned} t_{1}+\frac{46189}{131072} x_{0}^{10}+\frac{693}{256} x_{0}^{5} t_{11}+\frac{109395}{32768} x_{0}^{8} t_{17}+\frac{6435}{2048} x_{0}^{7} t_{15}+ \\
+\frac{3003}{1024} x_{0}^{6} t_{13}+\frac{15}{8} x_{0}^{2} t_{5}+\frac{3}{2} x_{0} t_{3}+\frac{315}{128} x_{0}^{4} t_{9}+\frac{35}{16} x_{0}^{3} t_{7}=0, \\
Y^{2}=\frac{1}{262144}\left(X-x_{0}\right)\left(256 X^{8} x_{0}-1024 X^{7} x_{0}^{2}-448 X^{6} x_{0}^{3}+\right. \\
+ \\
+672 X^{5} x_{0}^{4}+240 X^{4} x_{0}^{5}-160 X^{3} x_{0}^{6}- \\
\left.\quad-40 X^{2} x_{0}^{7}+10 X x_{0}^{8}+512 X^{9}+x_{0}^{9}\right)^{2}\end{aligned}$ \\
\hline
\end{tabular}




\begin{tabular}{|c|c|c|}
\hline$(2,21)$ & $\begin{array}{c}t_{3}=\frac{2476099}{1185408} \mu^{5} \\
t_{7}=\frac{1433531}{197568} \mu^{4} \\
t_{11}=\frac{89167}{10584} \mu^{3} \\
t_{15}=\frac{361}{84} \mu^{2} \\
\mu=-\frac{21}{76} x_{0}^{2}\end{array}$ & $\begin{aligned} & t_{1}+\frac{88179}{262144} x_{0}^{11}+\frac{109395}{32768} x_{0}^{8} t_{17}+\frac{230945}{65536} x_{0}^{9} t_{19}+ \\
&+\frac{35}{16} x_{0}^{3} t_{7}+\frac{315}{128} x_{0}^{4} t_{9}+\frac{6435}{2048} x_{0}^{7} t_{15}+ \\
&+\frac{693}{256} x_{0}^{5} t_{11}+\frac{3003}{1024} x_{0}^{6} t_{13}+\frac{15}{8} x_{0}^{2} t_{5}+\frac{3}{2} x_{0} t_{3}=0, \\
& Y^{2}=\frac{1}{1048576}\left(X-x_{0}\right)\left(512 X^{9} x_{0}-2304 X^{8} x_{0}^{2}-\right. \\
&-1024 X^{7} x_{0}^{3}+1792 X^{6} x_{0}^{4}+672 X^{5} x_{0}^{5}-560 X^{4} x_{0}^{6}- \\
&\left.-160 X^{3} x_{0}^{7}+60 X^{2} x_{0}^{8}+10 X x_{0}^{9}+1024 X^{10}-x_{0}^{10}\right)^{2}\end{aligned}$ \\
\hline$(2,23)$ & $\begin{array}{c}t_{1}=\frac{1801088541}{3295407616} \mu^{6} \\
t_{5}=\frac{943427331}{179098240} \mu^{5} \\
t_{9}=\frac{9270261}{778688} \mu^{4} \\
t_{13}=\frac{46305}{4232} \mu^{3} \\
t_{17}=\frac{441}{92} \mu^{2} \\
\mu=-\frac{23}{84} x_{0}^{2}\end{array}$ & $\begin{aligned} t_{1}+ & \frac{676039}{2097152} x_{0}^{12}+\frac{3003}{1024} x_{0}^{6} t_{13}+\frac{6435}{2048} x_{0}^{7} t_{15}+ \\
& +\frac{230945}{65536} x_{0}^{9} t_{19}+\frac{969969}{262144} x_{0}^{10} t_{21}+\frac{109395}{32768} x_{0}^{8} t_{17}+ \\
& +\frac{693}{256} x_{0}^{5} t_{11}+\frac{315}{128} x_{0}^{4} t_{9}+\frac{35}{16} x_{0}^{3} t_{7}+\frac{15}{8} x_{0}^{2} t_{5}+\frac{3}{2} x_{0} t_{3}=0, \\
Y^{2}= & \frac{1}{4194304}\left(X-x_{0}\right)\left(-x_{0}^{11}+2048 X^{11}+1024 x_{0} X^{10}-\right. \\
& -5120 x_{0}^{2} X^{9}-2304 x_{0}^{3} X^{8}+4608 x_{0}^{4} X^{7}+1792 x_{0}^{5} X^{6}- \\
& -1792 x_{0}^{6} X^{5}-560 x_{0}^{7} X^{4}+280 x_{0}^{8} X^{3}+60 x_{0}^{9} X^{2}- \\
& \left.-12 x_{0}^{10} X\right)^{2}\end{aligned}$ \\
\hline
\end{tabular}

Благодарности. Автор выражает благодарность А. В. Маршакову за полезные обсуждения и А. С. Анохиной за ценные замечания. Исследование выполнено при финансовой поддержке Министерства науки и образования РФ (соглашение 8498).

\section{Список литературы}

[1] G. 't Hooft, Nucl. Phys. B, 72:3 (1974), 461-473.

[2] А. В. Маршаков, ТМФ, 147:2 (2006), 163-228.

[3] I. M. Krichever, Commun. Pure Appl. Math., 47:4 (1994), 437-475, arXiv: hep-th/9205110.

[4] A. Gorsky, I. M. Krichever, A. Marshakov, A. Mironov, A. Morozov, Phys. Lett. B, 355:3-4 (1995), 466-474, arXiv: hep-th/9505035.

[5] A. Marshakov, Seiberg-Witten Theory and Integrable Systems, World Scientific, Singapore, 1999; "Seiberg-Witten curves and integrable systems", Integrability: The Seiberg-Witten and Whitham Equations, eds. H. W. Braden, I. M Krichever, Gordon and Breach, Amsterdam, 2000, 69-91.

[6] С. М. Харчев, А. В. Маршаков, А. Д. Миронов, А. Ю. Морозов, ТМФ, 95:2 (1993), 280-292, arXiv: hep-th/9208046.

[7] A. A. Migdal, Phys. Rep., 102:4 (1983), 199-290.

[8] V. A. Kazakov, Modern Phys. Lett. A, 4:22 (1989), 2125-2139; E. Brézin, V. A. Kazakov, Phys. Lett. B, 236:2 (1990), 144-150; M. R. Douglas, S. H. Shenker, Nucl. Phys. B, 335:3 (1990), 635-654; D. Gross, A. Migdal, Phys. Rev. Lett., 64:2 (1990), 127-130.

[9] Yu. Makeenko, A. Marshakov, A. Mironov, A. Morozov, Nucl. Phys. B, 356:3 (1991), 574-628.

[10] A. A. Belavin, A. M. Polyakov, A. B. Zamolodchikov, Nucl. Phys. B, 241:2 (1984), 333-380.

4 Теоретическая и математическая физика, т. 174, № 1, 2013 г. 
[11] A. M. Polyakov, Phys. Lett. B, 103:3 (1981), 207-210.

[12] N. Seiberg, D. Shih, JHEP, 02 (2004), 021, 61 pp., arXiv: hep-th/0312170; C. R. Physique, 6:2 (2005), 165-174, arXiv: hep-th/0409306.

[13] A. A. Belavin, A. B. Zamolodchikov, J. Phys. A, 42:30 (2009), 304004, 18 pp., arXiv: 0811.0450 .

[14] G. Moore, N. Seiberg, M. Staudacher, Nucl. Phys. B, 362:3 (1991), 665-709.

[15] Al. Zamolodchikov, Internat. J. Modern Phys. A, 19:supp02 (2004), 510-523, arXiv: hep-th/0312279.

[16] A. Marshakov, J. Phys. A, 42:30 (2009), 304021, 16 pp. 